\section{Disorders of Initiating and Maintaining}

\section{Sleep}

Helga Peter ${ }^{1}$ und Thomas Penzel ${ }^{2}$

${ }^{1}$ Marburg, Deutschland

${ }^{2}$ Interdisziplinäres Schlafmedizinisches Zentrum, Charité Universitätsmedizin Berlin, Berlin, Deutschland

\section{Synonyme}

DIMS; Ein- und Durchschlafstörungen

\section{Definition}

DIMS stellten die Hauptkategorie A im Klassifikationssystem der Schlafstörungen der ASDC von 1979 dar, welche die Hauptbeschwerden der Patienten zur Grundlage der Einteilung in vier Kategorien machte. Die drei weiteren Hauptkategorien waren als Gruppe B die Störungen mit exzessiver Schläfrigkeit ( $\triangleright$ „Disorders of Excessive Somnolence“, DOES), als Gruppe C die Störungen des Schlaf-WachRhythmus (Disorders of the Sleep-Wake Schedule) und als Gruppe D die Dysfunktionen in Verbindung mit Schlaf, Schlafstadien oder partiellem Erwachen, Parasomnien (Disorders of Sleep, Sleep Stages or Partial Arousals, Parasomnias).

Siehe auch $\triangleright$ „Diagnostische Klassifikationssysteme“. 